PROCEEDINGS OF THE

AMERICAN MATHEMATICAL SOCIETY

Volume 125, Number 4, April 1997, Pages 1089-1094

S 0002-9939(97)03685-X

\title{
WILSON'S FUNCTIONAL EQUATION FOR VECTOR AND MATRIX FUNCTIONS
}

\author{
PAVLOS SINOPOULOS
}

(Communicated by Palle E. T. Jorgensen)

\begin{abstract}
We determine the general solution of the functional equation

$$
f(x+y)+f(x-y)=A(y) f(x) \quad(x, y \in G),
$$

where $G$ is a 2-divisible abelian group, $f$ is a vector-valued function and $A$ is a matrix-valued function. Using this result we solve the scalar equation

$$
f(x+y)+f(x-y)=g_{1}(x) h_{1}(y)+g_{2}(x) h_{2}(y) \quad(x, y \in G),
$$

which contains as special cases, among others, the d'Alembert and Wilson equations and the parallelogram law.
\end{abstract}

\section{INTRODUCTION}

Let $G$ be an abelian group divisible by 2 (that is, $2 G=G$ ) and $K$ an algebraically closed commutative field of characteristic different from 2 and 3 . Throughout this paper we denote by $\mathcal{L}, \mathcal{M}, \mathcal{N}$ the sets of all solutions $f: G \rightarrow K$ of the functional equations $f(x+y)=f(x)+f(y), f(x+y)=f(x) f(y)$ and $f(x+y)+f(x-y)=$ $2 f(x)+2 f(y)$, respectively. Also, $K_{2}$ is the set of all $2 \times 2$ matrices over $K, K^{2}$ is the set of all $2 \times 1$ matrices (column vectors) over $K, \mathcal{F}$ is the set of all vector-valued functions with linearly independent components, $I$ is the unit matrix of $K_{2}$ and 0 (the zero of $K$ ) is also used for the zeros of $G, K^{2}$ and $K_{2}$. If $a \in \mathcal{L}$, then $a$ is called additive.

Clearly, if $M \in K_{2}, f \in \mathcal{F}$ and $M f=0$, then $M=0$.

In this paper we determine the general solutions $f: G \rightarrow K^{2}, A: G \rightarrow K_{2}$ of the functional equation

$$
f(x+y)+f(x-y)=A(y) f(x) \quad(x, y \in G),
$$

which can be viewed as a vector analogue of Wilson's functional equation (1.1) below (cf. also Eq. (3.4) of [4]).

The main result is given in Theorem 1. Using this result we solve in Theorem 2 the scalar equation

$$
f(x+y)+f(x-y)=g_{1}(x) h_{1}(y)+g_{2}(x) h_{2}(y) \quad(x, y \in G) .
$$

This last equation contains as special cases the Wilson equation

$$
f(x+y)+f(x-y)=f(x) g(y),
$$

Received by the editors August 4, 1995 and, in revised form, September 22, 1995.

1991 Mathematics Subject Classification. Primary 39B42, 39B52, 39B62.

(C)1997 American Mathematical Society 
introduced in [9], the equations

$$
\begin{gathered}
f(x+y)+f(x-y)=2 f(x)+g(x) h(y), \\
f(x+y)+f(x-y)=f(x) g(y)+g(x) f(y), \\
f(x+y)+f(x-y)=f(x) f(y)+g(x) g(y),
\end{gathered}
$$

which were solved in [1], [3], and the parallelogram law

$$
f(x+y)+f(x-y)=2 f(x)+2 f(y),
$$

which characterizes the diagonalization of biadditive functions ([1], [2]). More general equations were solved e.g. in [4], but, as remarked in [3], to extract the solutions of (1.1)-(1.5) from [4] is not simple.

\section{The RESUlts}

Theorem 1. If $A: G \rightarrow K_{2}, f: G \rightarrow K^{2}$ with $f \in \mathcal{F}$ is a solution of the functional equation

$$
f(x+y)+f(x-y)=A(y) f(x) \quad(x, y \in G)
$$

then

$$
\left\{\begin{array}{l}
A(y)=C[E(y)+E(-y)] C^{-1}, \\
f(x)=C[E(x) \alpha+E(-x) \beta],
\end{array}\right.
$$

where $C \in K_{2}(\operatorname{det} C \neq 0), \alpha, \beta \in K^{2}$ and $E(x)$ has one of the forms

$$
\begin{aligned}
& E(x)=\chi(x)\left\|\begin{array}{cc}
1 & \phi(x) \\
0 & 1
\end{array}\right\| \\
& \left\|\begin{array}{cc}
1 & n(x)+\phi(x) \\
0 & 1
\end{array}\right\|,\left\|\begin{array}{cc}
\phi(x)+1 & c \phi(x)^{3}+3 c \phi(x)^{2}+\phi_{1}(x) \\
0 & \phi(x)+1
\end{array}\right\|, \\
& \left\|\begin{array}{cc}
\chi_{1}(x)-\left[\chi_{1}(0)-1\right]\left[\phi_{1}(x)+1\right] & 0 \\
0 & \chi_{2}(x)-\left[\chi_{2}(0)-1\right]\left[\phi_{2}(x)+1\right]
\end{array}\right\|,
\end{aligned}
$$

with $c \in K, \phi, \phi_{1}, \phi_{2} \in \mathcal{L}, \chi, \chi_{1}, \chi_{2} \in \mathcal{M}, \chi(x) \not \equiv 0$, and $n \in \mathcal{N}$.

Conversely, any $A, f$ as described above satisfy (2.1).

Note. If $f \notin \mathcal{F}$, then (2.1) reduces to a system of two Wilson equations and the solutions are obtained by application of Theorem (2.2) of [5].

Proof. Setting $y=u+v$ and $y=u-v$ in (2.1), adding the resulting equations and using (2.1) again we obtain

$$
A(v) A(u) f(x)=[A(u+v)+A(u-v)] f(x) .
$$

Since $f \in \mathcal{F}$ this leads to the matrix d'Alembert equation

$$
A(v) A(u)=A(u+v)+A(u-v) .
$$

Replacing $y$ by $-y$ in (2.1) we see that $A(y) f(x)=A(-y) f(x)$ and so $A(y)=$ $A(-y)$. Hence, interchanging $u, v$ in $(2.4)$, we have $A(u) A(v)=A(v) A(u)$. Since $K$ is algebraically closed there exists an invertible matrix $C \in K_{2}$ such that the matrix function $B(x):=C^{-1} A(x) C$ has the form

$$
B(x)=\left\|\begin{array}{cc}
b_{1}(x) & b_{0}(x) \\
0 & b_{2}(x)
\end{array}\right\|
$$

with $b_{1}(x) \equiv b_{2}(x)$ or $b_{0}(x) \equiv 0$ (see [8], [4]). 
Let $g(x):=C^{-1} f(x)$. Substituting

$$
A(x)=C B(x) C^{-1} \quad \text { and } \quad f(x)=C g(x)
$$

into (2.1) we get

$$
g(x+y)+g(x-y)=B(y) g(x)
$$

that is, with $g=\left\|\begin{array}{l}g_{1} \\ g_{2}\end{array}\right\|$,

$$
\begin{gathered}
g_{1}(x+y)+g_{1}(x-y)=g_{1}(x) b_{1}(y)+g_{2}(x) b_{0}(y), \\
g_{2}(x+y)+g_{2}(x-y)=g_{2}(x) b_{2}(y) .
\end{gathered}
$$

Substituting also the first of (2.5) into (2.4) we have

$$
B(u+v)+B(u-v)=B(u) B(v),
$$

that is,

$$
\begin{aligned}
b_{i}(u+v)+b_{i}(u-v) & =b_{i}(u) b_{i}(v), \quad i=1,2, \\
b_{0}(u+v)+b_{0}(u-v) & =b_{1}(u) b_{0}(v)+b_{0}(u) b_{2}(v) .
\end{aligned}
$$

We distinguish three cases.

Case 1. $b_{1}(x) \equiv b_{2}(x) \not \equiv 2$. Then $b_{1}\left(x_{0}\right)=b_{2}\left(x_{0}\right) \neq 2$ for some $x_{0} \in G$. Setting $y=0$ in (2.6) gives $B(0)=2 I$ (since $g \in \mathcal{F}$ ) and from (2.7) it follows that $B(2 x)+2 I=B(x)^{2}$. So the matrix $B\left(2 x_{0}\right)-2 I=B\left(x_{0}\right)^{2}-4 I$ is invertible. This and all subsequent $2 \times 2$ matrices are upper triangular with equal diagonal elements and hence commute.

Now the solutions of the matrix equation (2.7) can be written in the form $B(x)=$ $\Xi(x)+\Xi(-x)$ where $\Xi(x)=\chi(x)\left\|\begin{array}{cc}1 & \phi(x) \\ 0 & 1\end{array}\right\|$ with $\phi \in \mathcal{L}, \chi \in \mathcal{M}$ and $\Xi(x+y)=$ $\Xi(x) \Xi(y)$ (see, e.g., the proof of Lemma 1 of [7]).

In order to solve the equation (2.6) we follow the method used in the proof of Theorem 2.2 of [5]. In (2.6) we replace $x$ by $-x$, we subtract the resulting equation from (2.6) and, setting $h(x)=g(x)-g(-x)$, we obtain

$$
h(x+y)+h(x-y)=B(y) h(x)
$$

with $h(0)=0, h(-x)=-h(x)$ and $h(2 x)=B(x) h(x)$. Therefore

$$
\begin{aligned}
& {[2 B(x+y)-B(x) B(y)] h(x)=2 B(x+y) h(x)-B(y) h(2 x)} \\
& \quad=2[h(2 x+y)-h(y)]-[h(2 x+y)+h(2 x-y)] \\
& \quad=h(y+2 x)+h(y-2 x)-2 h(y)=B(2 x) h(y)-2 h(y) .
\end{aligned}
$$

Since $2 B(x+y)-B(x) B(y)=[\Xi(x)-\Xi(-x)][\Xi(y)-\Xi(-y)]$ we have

$$
[B(2 x)-2 I] h(y)=[\Xi(x)-\Xi(-x)][\Xi(y)-\Xi(-y)] h(x) .
$$

Setting $x=x_{0}$ we obtain

$$
g(y)-g(-y)=[\Xi(y)-\Xi(-y)] \gamma
$$

where $\gamma \in K^{2}$. Adding this to $g(y)+g(-y)=[\Xi(y)+\Xi(-y)] g(0)$ (which follows from (2.6) with $x=0$ ) we obtain

$$
g(x)=\Xi(x) \alpha+\Xi(-x) \beta,
$$

where $\alpha, \beta \in K^{2}$. So by (2.5) we obtain (2.2) with the first form in (2.3). 
Case 2. $b_{1}(x) \equiv b_{2}(x) \equiv 2$. Then (2.7b) shows that $b_{0} \in \mathcal{N}$ and from (2.6b) (a Jensen equation) we have $g_{2}(x)=\phi_{2}(x)+\lambda$ with $\lambda \in K$ and $\phi_{2} \in \mathcal{L}$. Now (2.6a) becomes

$$
g_{1}(x+y)+g_{1}(x-y)=2 g_{1}(x)+\left[\phi_{2}(x)+\lambda\right] b_{0}(y) .
$$

If $\phi_{2}(x) \equiv 0$, then interchanging $x, y$ in (2.8) and subtracting we find

$$
g_{1}(x-y)-g_{1}(y-x)=\left[2 g_{1}(x)-\lambda b_{0}(x)\right]-\left[2 g_{1}(y)-\lambda b_{0}(y)\right],
$$

a Pexider equation which gives $2 g_{1}(x)-\lambda b_{0}(x)=2 \phi_{0}(x)+2 \mu\left(\mu \in K, \phi_{0} \in \mathcal{L}\right)$. Hence

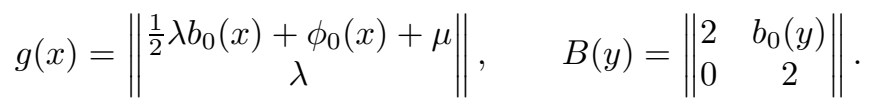

Using (2.5) we obtain (2.2) with the second form in (2.3).

If $\phi_{2}(x) \not \equiv 0$, then applying Lemma 4 of [1] to (2.8) (here we need the condition char $K \neq 2,3$ ) we obtain

$$
g_{1}(x)=c \phi_{2}(x)^{3}+3 c \lambda \phi_{2}(x)^{2}+\phi_{0}(x)+\mu, \quad b_{0}(y)=6 c \phi_{2}(y)^{2},
$$

where $c, \mu \in K$ and $\phi_{0} \in \mathcal{L}$. So by $(2.5)$ we obtain (2.2) with the third form in (2.3).

Case $3 . b_{0}(x) \equiv 0$. Then applying Theorem 2.2 of [5] to (2.6a) and (2.6b) we see that $g(x), B(y)$ have one of the forms

$$
\begin{array}{rlrl}
g(x) & =\left\|\begin{array}{ll}
\lambda \theta(x)+\mu \theta(-x) \\
\nu \eta(x)+\kappa \eta(-x)
\end{array}\right\|, & & B(y)=\left\|\begin{array}{cc}
\theta(y)+\theta(-y) & 0 \\
0 & \eta(y)+\eta(-y)
\end{array}\right\|, \\
g(x) & =\left\|\begin{array}{c}
a_{0}(x)+\lambda \\
\mu \theta(x)+\nu \theta(-x)
\end{array}\right\|, & B(y)=\left\|\begin{array}{ccc}
2 & 0 \\
0 & \theta(y)+\theta(-y)
\end{array}\right\|, \\
g(x)=\left\|\begin{array}{cl}
\mu \theta(x)+\nu \theta(-x) \\
a_{0}(x)+\lambda
\end{array}\right\|, & B(y)=\left\|\begin{array}{cc}
\theta(y)+\theta(-y) & 0 \\
0 & 2
\end{array}\right\|, \\
g(x)=\left\|\begin{array}{ll}
a_{0}(x)+\lambda \\
\phi_{0}(x)+\mu
\end{array}\right\|, & B(y) & =\left\|\begin{array}{ll}
2 & 0 \\
0 & 2
\end{array}\right\|,
\end{array}
$$

where $\theta, \eta \in \mathcal{M}, a_{0}, \phi_{0} \in \mathcal{L}$ and $\kappa, \lambda, \mu, \nu \in K$. So by (2.5) we obtain (2.2) with the last form in (2.3).

To prove the converse observe that the substitution of (2.2) into (2.1) gives

$$
\begin{aligned}
& {[E(x+y)+E(x-y)-[E(y)+E(-y)] E(x)] \alpha} \\
& +[E(-x+y)+E(-x-y)-[E(y)+E(-y)] E(-x)] \beta=0 .
\end{aligned}
$$

On the other hand, it is easy to verify that the functions $E$ in (2.3) satisfy the functional equation

$$
E(x+y)+E(x-y)=[E(y)+E(-y)] E(x) \quad(x, y \in G)
$$

and this completes the proof of Theorem 1.

In the following theorem the prime indicates transposition.

Theorem 2. If $f: G \rightarrow K, g, h: G \rightarrow K^{2}$ is a solution of the functional equation

$$
f(x+y)+f(x-y)=h^{\prime}(y) g(x) \quad(x, y \in G),
$$


then $f$ has the form

$$
f(x)=\lambda^{\prime}[E(x) \alpha+E(-x) \beta],
$$

where $\lambda, \alpha, \beta \in K^{2}$ and $E$ is given by (2.3).

Conversely, for any $f$ as described above there exist $g, h$ which together with $f$ constitute a solution of (2.10).

Notes. 1. Once $f$ is known, it is easy to determine the complete list for the vectorvalued functions $g, h$.

2. The right-hand side of (2.10), as a scalar product, can also be written $g^{\prime}(x) h(y)$.

Proof. If $g \notin \mathcal{F}$ or $h \notin \mathcal{F}$, then (2.10) reduces to a Wilson equation. Applying Theorem 2.2 of [5] to this equation we see that the solutions are contained in (2.11). So in the following we assume that $g, h \in \mathcal{F}$.

Setting $y=u+v$ and $y=u-v$ in (2.10), adding the resulting equations and using again (2.10) we find

$$
h^{\prime}(v)[g(x+u)+g(x-u)]=\left[h^{\prime}(u+v)+h^{\prime}(u-v)\right] g(x) .
$$

Since $h \in \mathcal{F}$, using Lemma 14.1 of [2] we obtain

$$
g(x+u)+g(x-u)=A(u) g(x),
$$

with $A: G \rightarrow K_{2}$. Since $g \in \mathcal{F}$, applying Theorem 1 to this equation we obtain

$$
g(x)=C[E(x) \alpha+E(-x) \beta)],
$$

where, $\alpha, \beta \in K^{2}, C \in K_{2}$ and $E$ is given by (2.3).

Now from (2.10) with $y=0$ we have $f(x)=\frac{1}{2} h^{\prime}(0) g(x)$ which leads to $(2.11)$.

The converse is verified by substitution of (2.11) into (2.10) and using (2.9).

Remark. The following result generalizes Theorem 1 and is proved in the same way. Here $S$ is an arbitrary set.

Theorem 3. If $A: G \rightarrow K_{2}, f: G \times S \rightarrow K^{2}$ with $f \in \mathcal{F}$ is a solution of the functional equation

$$
f(x+y, s)+f(x-y, s)=A(y) f(x, s) \quad(x, y \in G, s \in S),
$$

then

$$
\left\{\begin{array}{l}
A(y)=C\left[E\left(y, s_{0}\right)+E\left(-y, s_{0}\right)\right] C^{-1}, \\
f(x, s)=C[E(x, s) \alpha(s)+E(-x, s) \beta(s)],
\end{array}\right.
$$

where $s_{0} \in S, C \in K_{2}(\operatorname{det} C \neq 0), \alpha, \beta: S \rightarrow K^{2}$ are arbitrary functions and $E(x, s)$ has one of the forms

$$
\begin{aligned}
& E(x, s)=\chi(x)\left\|\begin{array}{cc}
1 & \phi(x) \\
0 & 1
\end{array}\right\|, \\
& \left.\left\|\begin{array}{ccc}
1 & n(x)+\phi_{1}(x, s) \\
0 & 1
\end{array}\right\|, \quad \| \begin{array}{cc}
\phi(x)+1 & c \phi(x)^{3}+3 c \phi(x)^{2}+\phi_{1}(x, s) \\
0 & \phi(x)+1
\end{array}\right], \\
& \left\|\begin{array}{cc}
\chi_{1}(x)-\left[\chi_{1}(0)-1\right]\left[\phi_{1}(x, s)+1\right] & 0 \\
0 & \chi_{2}(x)-\left[\chi_{2}(0)-1\right]\left[\phi_{2}(x, s)+1\right]
\end{array}\right\|,
\end{aligned}
$$

with $c \in K, \phi \in \mathcal{L}, \chi, \chi_{1}, \chi_{2} \in \mathcal{M}, \chi(x) \not \equiv 0, n \in \mathcal{N}$ and $\phi_{1}, \phi_{2}: G \times S \rightarrow K$ additive in the first variable.

Conversely, any A, $f$ as described above satisfy (2.12). 
(Clearly, for $E$ given by $(2.14)$, the sum $E(y, s)+E(-y, s)$ is independent of the second variable, that is, $E(y, s)+E(-y, s)=E(y, t)+E(-y, t)$ for all $y \in G$ and $s, t \in S$. So $A$ in the first of (2.13) is independent of the choice of $s_{0}$.)

Theorem 3 can be used, e.g., in the solution of the functional equation

$$
f(x+t, y-t)+f(x-t, y+t)=g_{1}(x, y) h_{1}(t)+g_{2}(x, y) h_{2}(t)
$$

which generalizes (2.10) (and (3.2) of [6]).

\section{ACKNOWLEDGMENT}

I wish to thank the referee for his valuable remarks which helped in improving this paper.

\section{REFERENCES}

1. J. Aczél, J. K. Chung and C. T. Ng, Symmetric second differences in product form on groups, Topics in mathematical analysis, (edited by Th. M. Rassias), World Scientific Publ., 1989, pp. 1-22. MR 92g:39007

2. J. Aczél and J. Dhombres, Functional equations in several variables, Cambridge Univ. Press, 1989. MR 90h:39001

3. J. K. Chung, Pl. Kannappan and C. T. Ng, On two trigonometric functional equations, Math. Rep. Toyama Univ. 11 (1988), 153-165. MR 89j:39010

4. A. L. Rukhin, The solution of the functional equation of d'Alembert's type for commutative groups, Intern. J. Math. Sci. 5 (1982), 315-335. MR 84g:39006

5. P. Sinopoulos, Generalized sine equations, I, Aeq. Mathematicae 48 (1994), 171-193. MR 95i:39020

6. P. Sinopoulos, Generalized sine equations, II, Aeq. Mathematicae 49 (1995), 122-152. MR 96b:39020

7. P. Sinopoulos, A functional equation in three variables for five unknown functions, Submitted.

8. D. A. Suprunenco and R. I. Tyshkevich, Commutative matrices, Academic Press, 1968.

9. W. H. Wilson, On certain related functional equations, Bull. Amer. Math. Soc. 26 (1919-20), 300-312.

18 Vergovitsas Street, GR-11475 Athens, Greece 